\title{
340-GHz Signal Source Design with Integrated On-Chip Bondwire Antenna in 40-nm CMOS
}

\author{
Te-Yen Chiu ${ }^{1}$, Chun-Lin $\mathrm{Ko}^{2}$, Chun-Hsing $\mathrm{Li}^{1+}$, Ming-Ching $\mathrm{Kuo}^{3}$, and Da-Chiang Chang ${ }^{2}$ \\ ${ }^{1}$ Department of Electrical Engineering, National Central University, Jhongly, Taiwan \\ ${ }^{2}$ National Chip Implementation Center, National Applied Research Laboratories, Hsinchu, Taiwan \\ ${ }^{3}$ Information and Communication Research Laboratories, Industrial Technology Research Institute, Hsinchu, \\ Taiwan
}

\begin{abstract}
This work presents two 340-GHz signal source designs with on-chip antennas for terahertz (THz) imaging applications. The first signal source $\left(1^{\text {st }} \mathrm{SS}\right)$ adopts a triple-push oscillator topology to generate a single-ended $\mathrm{THz}$ signal which is radiated out by a proposed on-chip high-gain bondwire antenna with simulated antenna gain as high as $2.9 \mathrm{dBi}$ at $340 \mathrm{GHz}$. The second signal source $\left(2^{\text {nd }} \mathrm{SS}\right)$ is realized by a proposed triple-push oscillator topology which can provide differential output without any additional balun required. The differential output is radiated to the air by an on-chip differential patch antenna with simulated antenna gain of $-3.9 \mathrm{dBi}$ at $340 \mathrm{GHz}$. These two signal sources are implemented in 40-nm digital CMOS technology. The measured output frequency and the equivalent isotropically radiated power of the $1^{\text {st }} \mathrm{SS}$ and $2^{\text {nd }} \mathrm{SS}$ are $346 \mathrm{GHz}$ and $336 \mathrm{GHz}$, and $-17.1 \mathrm{dBm}$ and $-16.8 \mathrm{dBm}$, while only consuming $58.3 \mathrm{~mW}$ and 44.6 $\mathrm{mW}$ from a $1.1 \mathrm{~V}$ supply, respectively.
\end{abstract}

Keywords: CMOS, signal source, on-chip antenna, THz, imaging system.

\section{Introduction}

Terahertz (THz) science and technology have attracted great attention in recent years because of many powerful applications [1-4]. THz imaging is especially appealing since it can be utilized to implement many useful sensors for non-invasive biomedical imaging, defect detection in semiconductors, dosage analysis of tablets in pharmaceutical industry, stand-off detection of hidden explosives and weapons, etc. Moreover, $\mathrm{THz}$ wave is very safe technology as compared to the X-ray due to its non-ionizing nature. Many $\mathrm{THz}$ products based on optical technology can be seen on the market [5-7]. However, these optical products are usually bulky and high-cost. Using an electronic approach, especially using integrated circuits and systems in CMOS technologies, is a good choice to solve the aforementioned drawbacks of the optical one. CMOS technologies can provide high integration of analog and digital circuits, small form factor for integrating $\mathrm{THz}$ systems on portable devices, and high yield for mass production. Among THz imaging sub-systems, a THz signal source is critical since it determines the maximum distance a $\mathrm{THz}$ imaging system can operate as the system sensitivity is given.

A satisfactory $\mathrm{THz}$ signal source needs to provide sufficient output power, usually characterized by equivalent isotropically radiated power (EIRP) that is defined as the product of the antenna gain $G_{\mathrm{T}}$ and the output power from a signal source $P_{\mathrm{T}}$. Since $P_{\mathrm{T}}$ is usually limited using CMOS technology because of limited transistor speed, lossy silicon substrate, etc, the antenna needs to provide high gain in order to achieve acceptable EIRP. However, on-chip antennas usually exhibit low gain due to lossy silicon substrate, short distance between the top metal layer to the bottom ground layer, and unavoidable metal dummies for passing design-rule checks. Therefore, the realized EIRP using CMOS technology is quite low. Combining

${ }^{+}$Corresponding author. Tel.: + 886034227151 Ext: 34516; Fax: +886034255830.

E-mail address: chli@ee.ncu.edu.tw 
many signal sources indeed raises the EIRP. Nevertheless, the power consumption also increases dramatically, which is not suitable for portable applications.

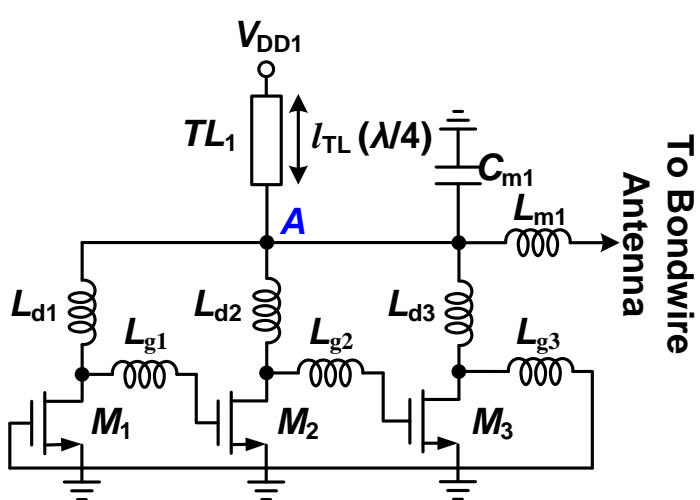

(a)

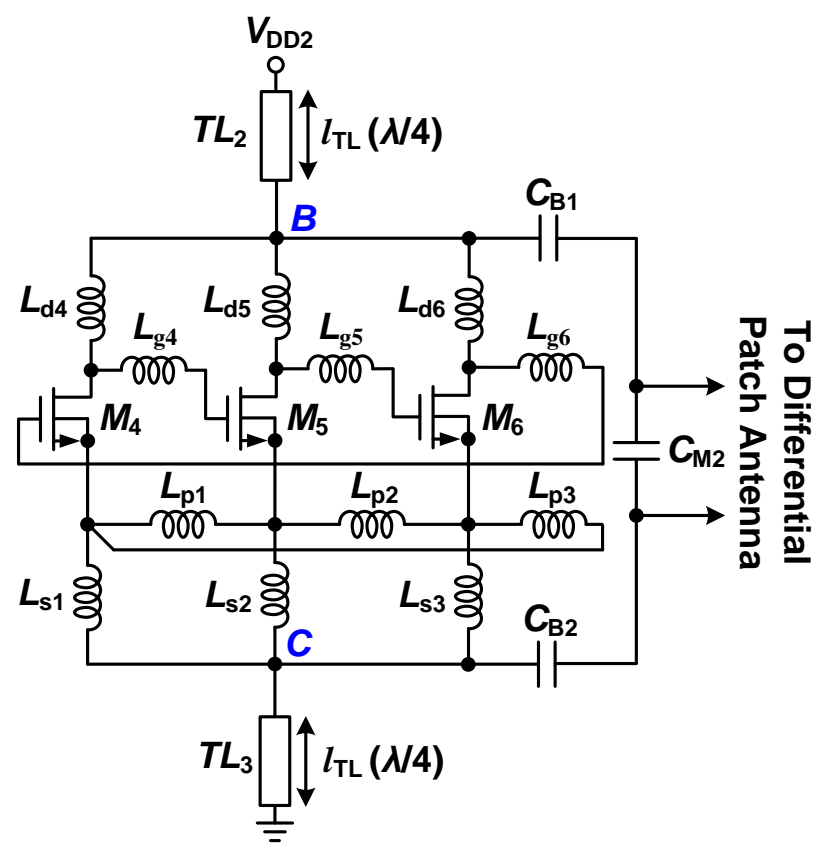

(b)

Fig. 1. Triple-push oscillator designs with (a) single-ended output and (b) differential output.
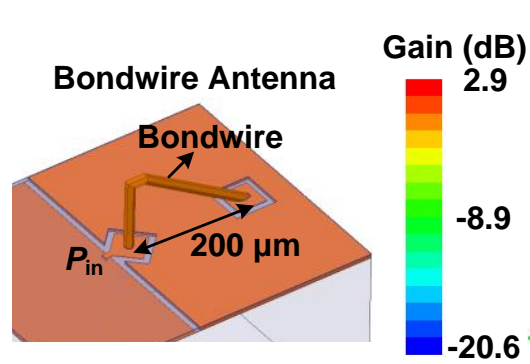

(a)
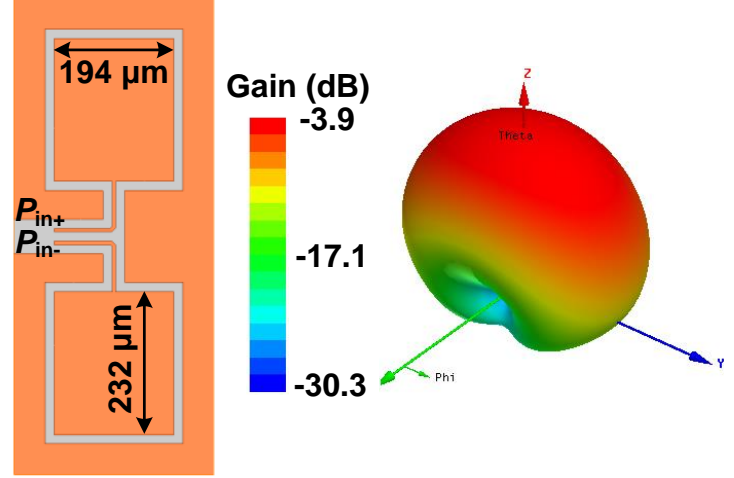

(b)

Fig. 2. Proposed on-chip antennas. (a) Bondwire antenna. (b) Differential patch antenna.

In this work, two THz signal sources are proposed with integrated on-chip antennas. The first signal source $\left(1^{\text {st }} \mathrm{SS}\right)$ adopts a triple-push oscillator topology to generate a $\mathrm{THz}$ signal with single-ended output which is radiated out by a proposed bondwire antenna. The bondwire antenna can provide simulated antenna gain of $2.9 \mathrm{dBi}$ at $340 \mathrm{GHz}$, which is much higher than a conventional on-chip patch antenna. The second source $\left(2^{\text {nd }} \mathrm{SS}\right)$ adopts a proposed triple-push oscillator which can provide differential output without any additional lossy balun required. Hence the output power from the signal source can be effectively increased. The differential output is radiated by a differential patch antenna which is composed a $1 \times 2$ patch antenna array deployed in a differentially-excited manner. The differential antenna can provide $-3.9 \mathrm{dBi}$ antenna gain at $340 \mathrm{GHz}$, which is around $3 \mathrm{~dB}$, as expected, higher than that of a single patch. These signal sources are realized in 40-nm digital CMOS technology. The measured output frequency and the EIRP of the $1^{\text {st }} \mathrm{SS}$ and the $2^{\text {nd }} \mathrm{SS}$ are $346 \mathrm{GHz}$ and $336 \mathrm{GHz}$, and $-17.1 \mathrm{dBm}$ and $-16.8 \mathrm{dBm}$, while dissipating only $58.3 \mathrm{~mW}$ and $44.6 \mathrm{~mW}$ from a $1.1 \mathrm{~V}$ supply, respectively. Theses signal sources are very suitable for realizing a low-cost $\mathrm{THz}$ imaging system for biomedical and security applications. The following sections will go through the design detail of the proposed signal sources. 


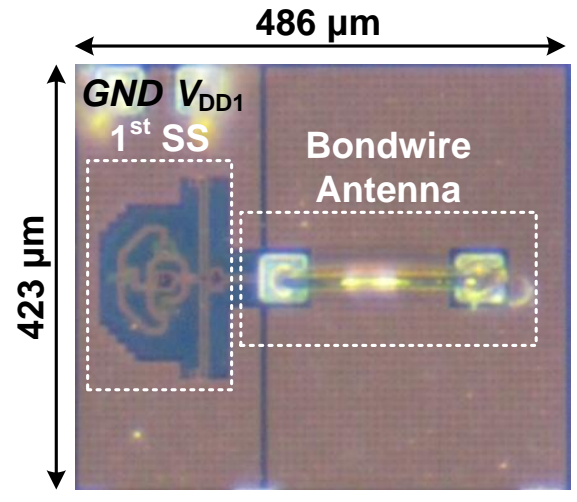

(a)

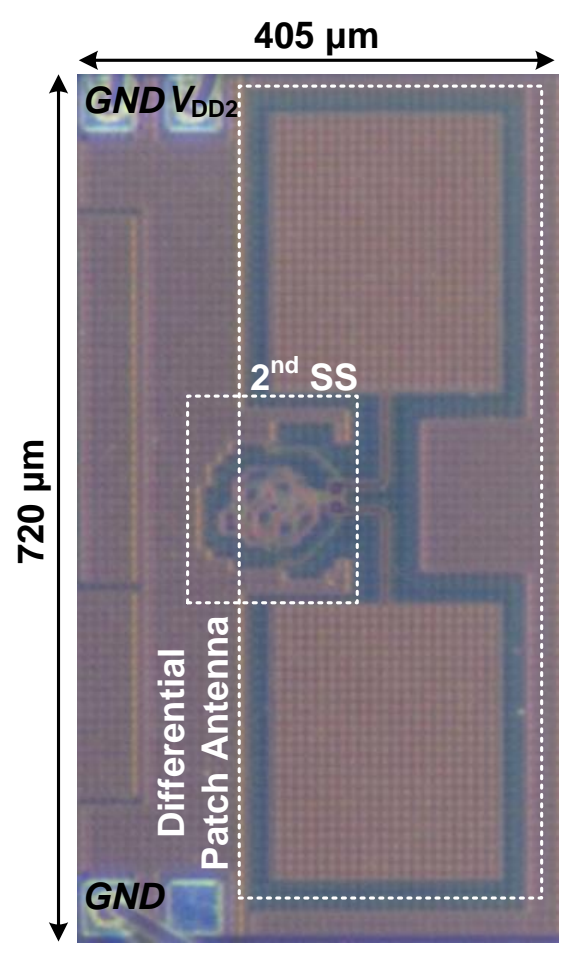

(b)

Fig. 3. Chip photos of (a) the $1^{\text {st }} \mathrm{SS}$ with the proposed on-chip bondwire antenna and (b) the $2^{\text {nd }} \mathrm{SS}$ with the proposed differential patch antenna.

\section{340-GHz Signal Source Designs with On-Chip Antennas}

\subsection{Triple-push oscillator design}

Fig. 1 shows the proposed two triple-push oscillator designs. As illustrated in Fig. 1(a), the first signal source adopts a conventional triple-push oscillator topology [8]. Essentially, it is a ring oscillator with a fundamental oscillation frequency of $f_{1}$. As the oscillator reaches a steady state, the voltages at the drain of each transistor have same amplitude but phase different of $120^{\circ}$. After combining these signals by inductors of $L_{\mathrm{d} 1-\mathrm{d} 3}$ at the node $A$, the third harmonics are added in phase while the fundamental and the second harmonics are cancelled. Therefore, the oscillator can generate an output signal with frequency of $f_{\text {osc }}$ equal to $3 f_{1}$ which can be easily designed to be higher than the transistor maximum oscillator frequency $f_{\max }$. $T L_{1}$ functions as a RF choke to provide a supply voltage to the oscillator while blocking an ac signal. $L_{\mathrm{M} 1}$ and $C_{\mathrm{M} 1}$ are employed to conduct a power matching between the oscillator and an antenna. The output signal is then radiated to the air by a proposed bondwire antenna which will be discussed in the next section. The oscillator is designed to have output frequency of $340 \mathrm{GHz}$ by proper selection of the inductance values of $L_{\mathrm{d} 1-\mathrm{d} 3}$ and $L_{\mathrm{g} 1-\mathrm{g} 3}$.

The second signal source, as shown in Fig. 1(b), adopts a proposed triple-push oscillator topology which can provide differential output without any additional balun required [9]. The oscillator not only extracts the output signals from the drain nodes, but also from the source nodes. By doing this, the third harmonics are constructively added at the nodes $B$ and $C$ simultaneously. Moreover, the voltage signals at the nodes of $B$ and $C$ have exactly same amplitude but $180^{\circ}$ phase difference. Such perfect differential output is due to the fact that the output signals are actually extracted by the same current loop, which implies the output currents have the same amplitude but out of phase. Since the output loads are the same, naturally the outputs are in a perfect differential form. This oscillator is also designed to have oscillation frequency of $340 \mathrm{GHz}$ by proper selection of the inductance values of $L_{\mathrm{d} 4-\mathrm{d} 6}, L_{\mathrm{g} 4-\mathrm{g} 6}, L_{\mathrm{s} 1-\mathrm{s} 3}$, and $L_{\mathrm{p} 1-\mathrm{p} 3} . T L_{2}$ and $T L_{3}$ work as $R F$ chokes to provide a supply voltage and the ground to the oscillator, respectively, while blocking ac signals. The output impedance is matched to $100 \Omega$ simply by adding a capacitor $C_{\mathrm{M} 2}$. The differential output signal is then radiated to the air by a differential patch antenna which will be discussed in the next section. 


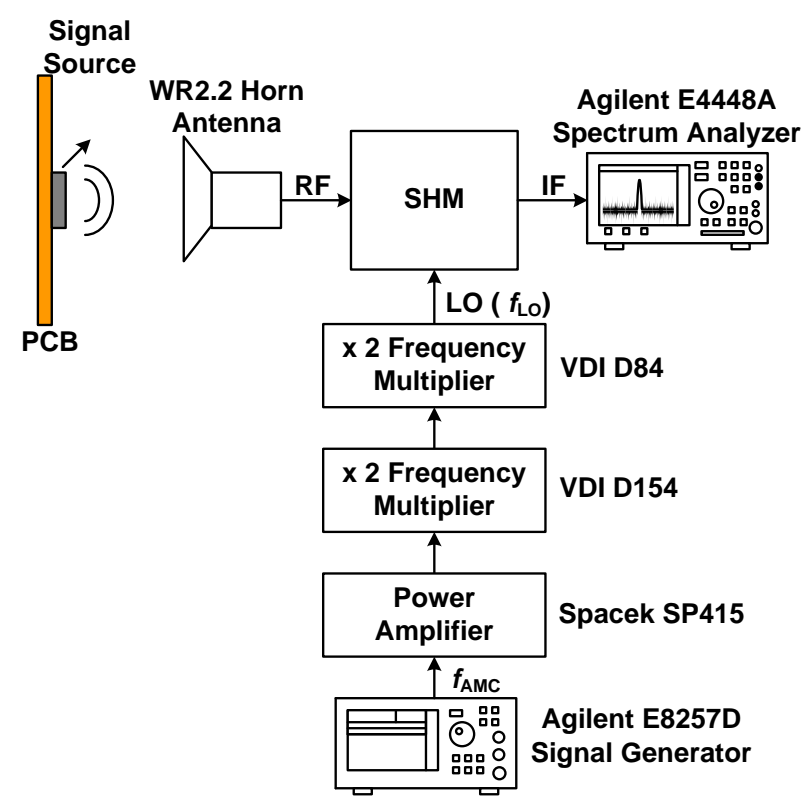

(a)

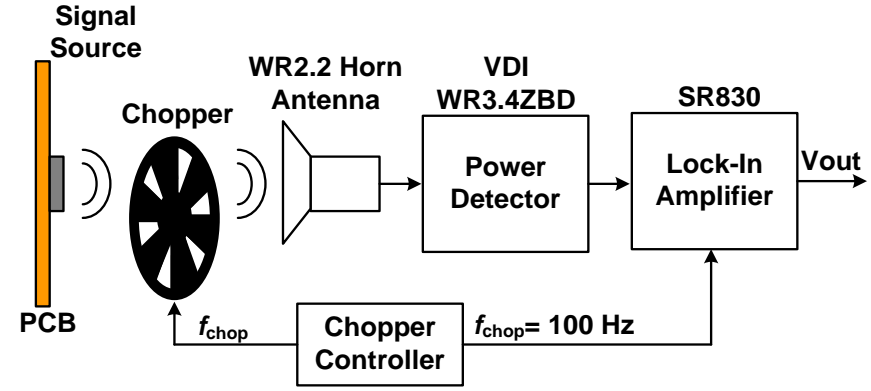

(b)

Fig. 4. Experimental setups for the measurement of (a) the oscillation frequency and (b) the EIRP.
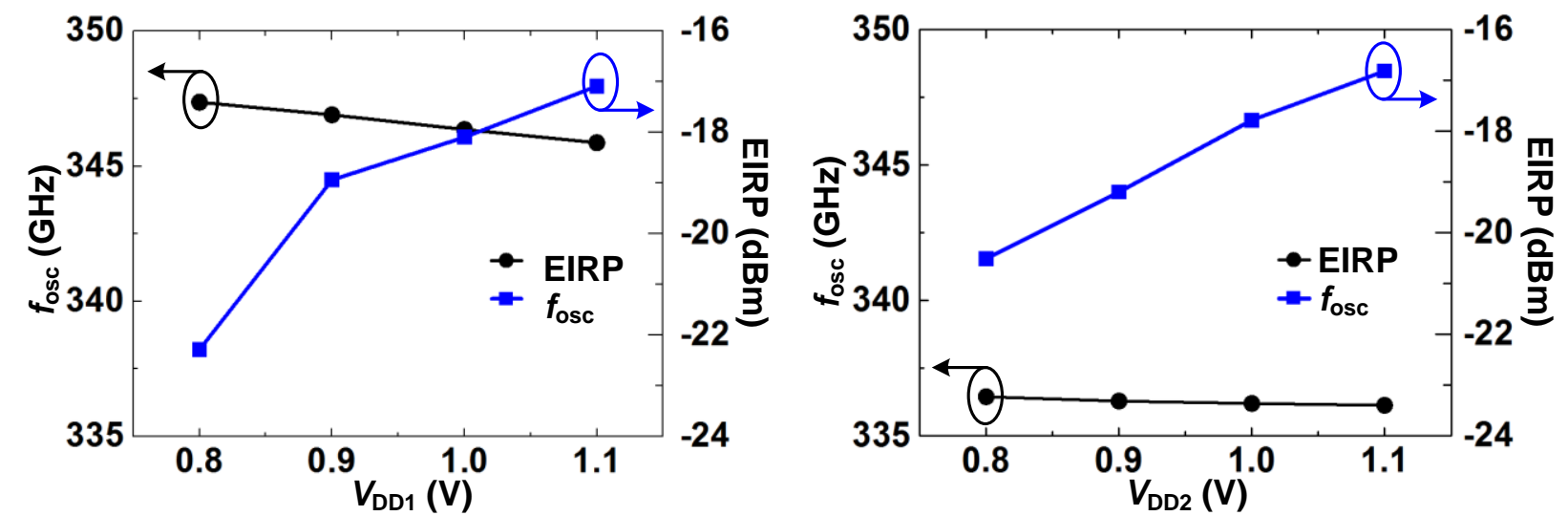

Fig. 5. Measured $f_{\text {osc }}$ and EIRP of (a) the $1^{\text {st }}$ SS with a bondwire antenna and (b) the $2^{\text {nd }}$ SS with a differential patch antenna.

\subsection{On-chip antenna design}

Fig. 2(a) illustrates the proposed bondwire antenna which is used to radiate the output signal from the $1^{\text {st }}$ SS. The antenna is composed of two bonding pads and a gold bondwire. By proper selection of the bondwire length, the antenna can provide antenna gain of $2.9 \mathrm{dBi}$ at $340 \mathrm{GHz}$ with the radiation pattern also shown in the Fig. 2(a). Such a high-gain antenna can solve the low-gain issue occurred in conventional on-chip antennas. The antenna input is conjugated matched to the $1^{\text {st }} \mathrm{SS}$ by $L_{\mathrm{M} 1}$ and $C_{\mathrm{M} 1}$ as depicted in Fig. 1(a).

The proposed differential patch antenna for radiating the output signal from the $2^{\text {nd }} \mathrm{SS}$ is shown in Fig. 2(b). It is composed of two patch antennas arranged in a differentially-excited manner. By injecting differential signals on the opposite of the patch antennas, the excited fields become in phase. Hence the antenna gain can be increased around $3 \mathrm{~dB}$ as compared with a single patch antenna. The input impedance is designed to have $100 \Omega$ differentially, which is matched to the $2^{\text {nd }}$ SS for the maximum power transfer.

\section{Experimental Results}

The proposed two signal sources are implemented in 40-nm digital CMOS technology without ultrathick metal (UTM) layers, metal-insulator-metal (MIM) capacitors, and metal-oxide-metal (MOM) capacitors available. The chip micrographs are shown in Fig. 3. The $1^{\text {st }} \mathrm{SS}$ and the $2^{\text {nd }} \mathrm{SS}$ occupy chip areas of $423 \mu \mathrm{m} \times 486 \mu \mathrm{m}$ and $405 \mu \mathrm{m} \times 720 \mu \mathrm{m}$, including the oscillators, antennas, and dc pads, respectively. The signal sources are characterized in the free space with the experimental setup illustrated in Fig. 4 . The oscillation frequency is measured using a subharmonic mixer (SHM) as indicated in Fig. 4(a). By observing 
the measured IF spectrum, the oscillation frequency $f_{\text {osc }}$ can be determined by $f_{\mathrm{IF}}+2 f_{\mathrm{LO}}$ where $f_{\mathrm{IF}}$ and $f_{\mathrm{LO}}$ are the measured IF frequency and the injected LO frequency to the SHM, respectively. The EIRP is measured using a power detector with a model number of WR3.4 ZBD from Virginia Diode Inc. (VDI). Since the output voltage of the power detector is low, the locking amplifier technique is used to detect such a small signal while rejecting undesired noise.

The measured $f_{\text {osc }}$ and the EIRP of these two signal sources are shown in Fig. 5 . Fort the $1^{\text {st }} \mathrm{SS}$, the oscillation frequency can be tuned from $347.4 \mathrm{GHz}$ to $345.9 \mathrm{GHz}$ while providing EIRP from $-22.3 \mathrm{dBm}$ to $17.1 \mathrm{dBm}$ as the supply is changed from $0.8 \mathrm{~V}$ to $1.1 \mathrm{~V}$. The $1^{\text {st }} \mathrm{SS}$ only consumes $58.3 \mathrm{~mW}$ from a $1.1 \mathrm{~V}$ supply. For the $2^{\text {nd }} \mathrm{SS}$, the oscillation frequency can be tuned from $336.4 \mathrm{GHz}$ to $336.1 \mathrm{GHz}$ while providing EIRP from $-20.5 \mathrm{dBm}$ to $-16.8 \mathrm{dBm}$ as the supply is changed from $0.8 \mathrm{~V}$ to $1.1 \mathrm{~V}$. The $2^{\text {nd }} \mathrm{SS}$ only consumes $44.6 \mathrm{~mW}$ from a $1.1 \mathrm{~V}$ supply. The proposed signal sources are very suitable for low-cost $\mathrm{THz}$ imaging applications.

\section{Conclusion}

Two signal sources with integrated on-chip antennas are proposed and demonstrated using 40-nm digital CMOS technology. The first signal sources provide single-ended output which is radiated to the air by a proposed high-gain on-chip bondwire antenna. The second signal source can provide differential output without additional lossy balun required. The differential output is radiated out by a differential patch antenna which is composed of two patch antennas arranged in a differentially-excited manner. The measured oscillation frequency and EIRP of the $1^{\text {st }} \mathrm{SS}$ and the $2^{\text {nd }} \mathrm{SS}$ are $346 \mathrm{GHz}$ and $336 \mathrm{GHz}$, and $-17.1 \mathrm{dBm}$ and $16.8 \mathrm{dBm}$, while only consuming $58.3 \mathrm{~mW}$ and $44.6 \mathrm{~mW}$ from a $1.1 \mathrm{~V}$ supply, respectively. These two signal sources are very suitable for low-cost $\mathrm{THz}$ imaging applications.

\section{Acknowledgements}

This work was funded by the MOST, under Grant MOST 103-2221-E-008-008-MY2. The authors would like to acknowledge the National Center for High-performance Computing for the ANSYS HFSS tool support, and Mr. Wei-Cheng Chen and Prof. Chien-Nan Kuo at Department of Electronics Engineering, National Chiao Tung University, Hsinchu, Taiwan, for THz measurement support.

\section{References}

[1] P. H. Siegel, “Terahertz technology," IEEE Trans. Microw. Theory Techn., vol. 50, no. 3, pp. 910-928, Mar. 2002.

[2] P. H. Siegel, "Terahertz technology in biology and medicine," IEEE Trans. Microw. Theory Techn., vol. 52, no. 10, pp. 2438-2447, Oct. 2004.

[3] M. Tonouchi, "Cutting-edge terahertz technology," Nature Photonics, vol. 1, pp. 97-105, Feb. 2007.

[4] H.-J. Song and T. Nagatsuma, "Present and future of terahertz communications," IEEE Trans. THz Sci. Technol., vol. 1, no. 1, pp. 256-263, Sep. 2011.

[5] TeraView, TeraPulse 4000 [Online]. Available: http://www.teraview.com/products/TeraPulse\%204000/index.html

[6] MenloSystems, TERA K15 [Online]. Available: http://www.menlosystems.com/products/thz-time-domainsolutions/all-fiber-coupled-terahertz-spectrometer/

[7] ADVANTEST, TAS7500TS [Online]. Available: https://www.advantest.com/products/terahertz-spectroscopicimaging-systems/terahertz-wave-spectroscopy-and-imaging-analysis-platform

[8] O. Momeni and E. Afshari, "High power terahertz and millimeter-wave oscillator design: A systematic approach," IEEE J. Solid-State Circuits, vol. 46, no. 3, pp. 583-597, Mar. 2011.

[9] C.-H. Li, C.-L. Ko, C.-N. Kuo, M.-C. Kuo, and D.-C. Chang, "A 340-GHz triple-push oscillator with differential output in 40-nm CMOS," IEEE Microw. Compon. Lett., vol. 24, no. 12, pp. 863-865, Dec. 2014. 